\title{
Quantum photonics based on metasurfaces
}

\author{
Jun Liu, Mingqian Shi, Zhuo Chen, Shuming Wang*, Zhenlin Wang* and \\ Shining Zhu*
}

From metamaterials to metasurfaces, optical nano-structure has been widely investigated for novel and high efficiency functionalities. Apart from the intrisinsic properties of composite material, rich capabilities can be derived from the judicious design of metasurfaces, which enable more excellent and highly integrated optical devices than traditional bulk optical elements. In the meantime, the abundant manipulation abilites of light in the classical domain can be carried over into quantum domain. In this review, we highlight recent development of quantum optics based on metasurfaces, ranging from quantum plasmonics, generation, manipulation and appplication of quantum light to quantum vaccum engineering etc. Finally, some promising avenues for quantum optics with the help of optical metasurface are presented.

Keywords: metasurfaces; quantum optics; quantum information

Liu J, Shi MQ, Chen Z, Wang SM, Wang ZL et al. Quantum photonics based on metasurfaces. Opto-Electron Adv 4, 200092 (2021).

\section{Introduction}

Optical metamaterial is composed of periodically arranged nano-optical elements whose size is much smaller than the working wavelength ${ }^{1-3}$. The special optical properties of metamaterials not only derive from its intrinsic material composition, but also can be manipulated by controlling its geometrical parameters, for example, shape, direction, arrangement and other degrees of freedom. Compared with conventional material, optical material has the capability of tailoring electromagnetic response at the subwavelength scale, rendering many exotic properties. On the one hand, man-made material which doesn't exist in nature with desired permeability or permittivity can be produced, and one of these categories is negative refractive index material ${ }^{1}$. On the other hand, the possibility of tailoring electromagnetic response at the subwavelength scale has made a wide range of novel devices available, such as superlenses ${ }^{4}$, hyperlenses $^{5,6}$, optical cloaking ${ }^{7,8}$ and so on. However, it's a pity that the fabrication of optical metamaterials is severely restricted nowadays, the current fabrication methods mainly consist of electron-beam lithography, focused ion beam lithography, nanoimprint lithography and directed self-assembly. It's not always easy to fabricate intricate microstructures that satisfy specific demands, hence, this greatly hinders the developments and applications of optical metamaterials. Interestingly, optical metasurfaces, a kind of artificial flake material with sub-wavelength thickness, can circumvent this problem effortlessly ${ }^{9}$. In fact, the optical metasurface is the twodimensional counterpart of metamaterials, and it can be either periodic or aperiodic. Although one dimension of space of the nano-patterned film is reduced, the truncated volume doesn't lower the efficiency to control phase, amplitude and polarization of light at will, and this indeed enables higher integration density and less processing difficulties through the same nano-fabrication technologies. Apart from this advantage, subwavelength thickness means reduced ohmic loss that is unavoidably associated with metal materials. Moreover,

National Laboratory of Solid-State Microstructures, Department of Physics, Nanjing University, Nanjing 210093, China.

"Correspondence: SM Wang, E-mail: wangshuming@nju.edu.cn; ZL Wang, E-mail: zlwang@nju.edu.cn; SL Zhu, E-mail: zhusn@nju.edu.cn Received: 14 December 2020; Accepted: 10 February 2021; Published: 25 September 2021 
it is worth mentioning that for nonlinear optics, the phase-matching condition can be significantly mitigated owing to the condensed thickness ${ }^{10-16}$.

When it comes to the manipulation of light for optical metasurfaces, generally speaking, there are two phase control approaches for optical metasurface: resonant phase and geometric phase, or called PancharatnamBerry phase ${ }^{17}$, the former depends on incident wavelength, size and shape of meta-atoms, and the latter only relies on the orientation of meta-atoms. In terms of material composition, metasurface can also be roughly divided into two types: metallic metasurface and dielectric metasurface, the former relies on surface plasmon polaritons $\mathrm{s}^{18}$, featuring huge local field enhancement $^{11,12,19-28}$, and the latter relies on Mie resonance ${ }^{14,29-38}$, although it generates relatively weaker local electric field, the interaction contributed by the magnetic component of incident light can't be ignored any more. Besides, thermal heating as well as dissipative losses is greatly reduced for nanostructures so as to withstand higher pump intensity.

Look back over the past few decades, the research fields of metasurfaces have primarily been focused on classical regime, and enormous efforts consider the classical behavior of light, including abnormal reflection and transmission 9 , planar lens imaging ${ }^{39,40}$, holography $y^{41-44}$, photon spin hall effect ${ }^{45}$ and vortex beam generation ${ }^{46,47}$, etc. However, one important thing that must be made clear is that any behavior of light is quantum mechanical, although we have created a broad range of highly integrated, efficient optical elements only in the viewpoint of classical physics. This is definitely not enough, and much less attention has been given to the quantum optics based on optical metasurface. As a matter of fact, optical metasurface is qualified to further upgrade the multifunctional integration capability of quantum optical technology. And nowadays in the domain of quantum optics, more and more efforts have been given to the advancement and development of integrated photonic quantum technologies rather than the breakthrough of basic principles of quantum mechanics. Quantum information ${ }^{48-53}$, quantum computation ${ }^{54-60}$ and quantum metrology ${ }^{61,62}$ are the main research hotspots, which can revolutionize current technology and human lives again. Otherwise some other photonic physical systems, such as superconductors ${ }^{63}$, cold atoms $s^{64}$, ions ${ }^{65}$, have been investigated fruitfully and show good performance in some way. Quantum optics based on metasurfaces is an emer- ging avenue for highly integrated quantum technologies. Not only can we fully exploit the integration and efficiency of metasurfaces to create robust, scalable and miniaturized quantum optical devices, but also unknown new physics may be discovered in the context of optical metasurface regime.

In this review, we highlight recent progress in the field of quantum optics based on metasurfaces. The review is organized as follows: we start by introducing the quantum optical effects based on surface plasmon polaritons (SPPs), we then review quantum light source based on the metasurface, manipulation of quantum state based on metasurfaces, quantum applications based on metasurfaces, and quantum vacuum engineering of quantum emitter through metasurfaces, finally we briefly discuss the future outlook of quantum optics with metasurfaces. For readers who want to know more about this topic, some other relevant reviews are recommended ${ }^{66-69}$.

\section{Quantum plasmonics}

As everyone knows, bosons and fermions are the only two types of fundamental particle in nature, they obey different statistics, and display distinguishable quantum behavior, such as coalescence or anti-coalescence for bosons and fermions respectively. For instance, photons are bosons and electrons are fermions. Something interesting happens when light is interacting with metallic nanostructure, photons couple with the free electrons on the surface of metal to generate surface plasmon polaritons (SPPs), which can be regarded as boson-like particles. So SPPs can not only provide huge local field enhancement but also inherit the quantum properties of photon rather than electron ${ }^{70,71}$. According to this, the applications with SPPs has quickly been carried over into quantum photonics. Interestingly, even if SPPs include the interaction and movement of massive electrons, under certain conditions, quantum behavior has been maintained. $\mathrm{Al}$ tewischer firstly observed the quantum entanglement of surface plasmon polaritons, and it was experimentally demonstrated that entanglement photon pairs through $\mathrm{Au}$ film metasurfaces still kept its quantum property ${ }^{72}$. Moreno employed quantum theory to explain this phenomenon ${ }^{73}$. Moreover, Fasel pointed out that photonSPP-photon conversion process kept the energy-time entanglement ${ }^{74}$. Huck experimentally confirmed the robustness of continuous variable quantum state in the propagation of SPPs ${ }^{75}$. Tan observed the quantum tunneling phenomenon between two adjacent SPPs 
resonators ${ }^{76}$. Kolesov discovered that single photon source coupled with Ag nanowire could excite the SPPs monopole, which had been inferred the wave-particle dualism alike to single photon ${ }^{77}$. Dheur judiciously designed SPP beam splitter to observe the self-interference of monopole, which confirmed the quantum inference ${ }^{78}$.

\section{Quantum sources}

Quantum entanglement and quantum interference are the two key concepts of quantum physics that differ from classical physics. They are indeed the driving force behind quantum technologies, such as quantum communication, quantum computation, quantum metrology and quantum sensing. To employ the unique physical phenomenon for advancing the development of quantum photonic technologies, the way to acquire large-area, stable, controllable and small-scale multiphoton high dimensional quantum source is highly desirable. On the one hand, increasing the dimension of quantum source can reveal more novel quantum physics embedded in nature. On the other hand, high dimension guarantees bigger information capacity and stronger resilience to environment noise in quantum communication regime. Moreover, compared with low dimensional quantum source, high dimensional quantum source offers higher efficiency and flexibility in quantum computation.

In recent years, researchers have demonstrated high dimensional quantum source using various degrees of freedom, such as spatial mode, energy-time, polarization, path and so on ${ }^{79}$. Utilizing a $N \times N$ multi-channel that splits a pump beam into $N$ coherent beams can create path-entangled quantum states ${ }^{80}$. And the way to align $N$ nonlinear crystals and insert spiral phase plate in between is able to prepare orbital angular momentum entangled quantum states through path identity ${ }^{81}$. There are also other related works based on on-chip photonics. Multidimensional integrated quantum photonic platform has been demonstrated that can generate, control, and analyze high-dimensional entanglement quantum states. This device consists of a total of 16 identical spiral waveguide sources based on SFWM and a total of more than 550 optical components, which provides reliable experimental platform for multidimensional quantum technologies ${ }^{82}$. Compared with aforementioned methods, optical metasurface possesses the merit of richer manipulation of light and small scale, which is very suitable for highly integrated quantum circuits. The following is mainly about quantum source based on optical metasurfaces.

Dependent on material selection, geometry control and rotation associated with meta-atoms, optical metamaterial provides abundant freedoms to manipulate the spontaneous parametric down conversion, having potential applicability for preparing desired multiphoton quantum state. According to this, the most universal method to produce entangled photon pair at will is intrinsically available in metamaterials ${ }^{83}$. Initially, theoretical prediction for generation of photon-plasmon pair is given, after taking loss and dispersion into consideration, $70 \%$ internal heralding quantum efficiency is achieved in layered metal-dielectric structures ${ }^{84}$. Of course, this method can be expanded to any other types of nanostructures too. Experimentally, some material, such as crystalline AlGaAs, is able to combine resonance enhancement with high nonlinear susceptibility togeth$\mathrm{er}^{85}$, thus bridging the gap between nonlinear optics and quantum optics. Over the past few decades, there has been so many works concerning how to augment and control nonlinear frequency conversion, and these works provide enormous and rich knowledge for manipulation of quantum source. In fact, 5 times enhancement of SPDC efficiency is attainable from subwavelength films made of $\mathrm{GaP}^{86}$, the enhancement originates from FabryPerot resonance inside the nonlinear films. Take it further, Y. Ming et al. theoretically demonstrated that by judiciously designing metamaterials, desired quantum state could be prepared from metamaterials ${ }^{87}$. However, as a consequence of fabrication difficulties and low efficiency of spontaneous parametric down conversion linked to three-dimensional metamaterial, quantum source based on metamaterial is still far away from practical application. To solve this problem, L. Li et al. demonstrated that by creatively combining optical metasurface and nonlinear crystal, high dimensional multiphoton quantum source is attainable ${ }^{88}$. As shown in Fig. 1(a), by putting the metasurface closely to nonlinear crystal and focusing the incident laser inside the center of nonlinear crystal, photon-pair is produced from the focus spot of individual metalens. When only one photon pair is generated, the path of photon pair cannot be distinguished, therefore path-entangled photon pair quantum state is prepared. For example, when three or four metalenses are selected to produce three-dimensional or four-dimensional photon-pair quantum state, 
a

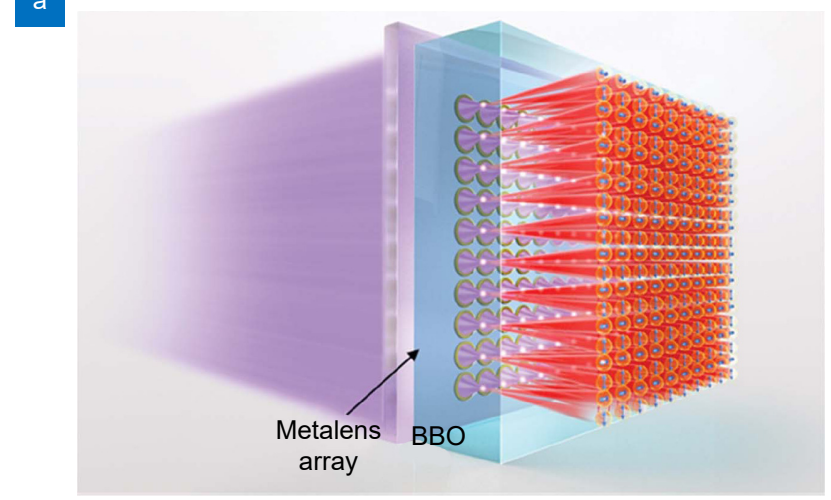

c
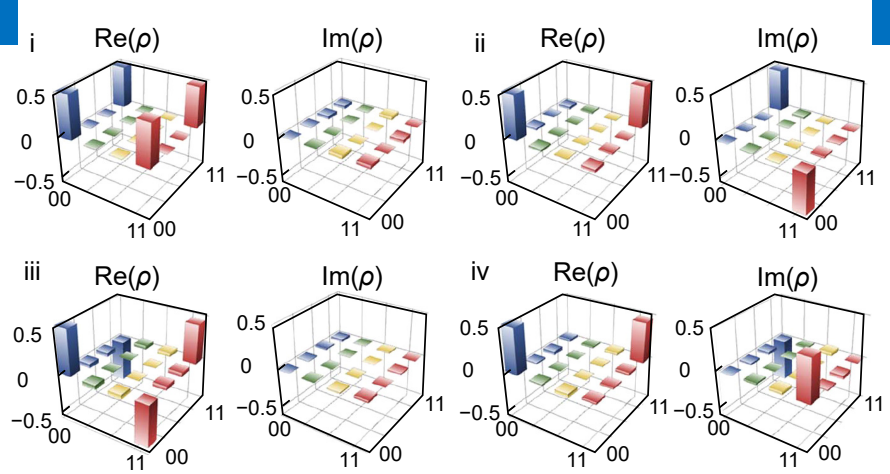
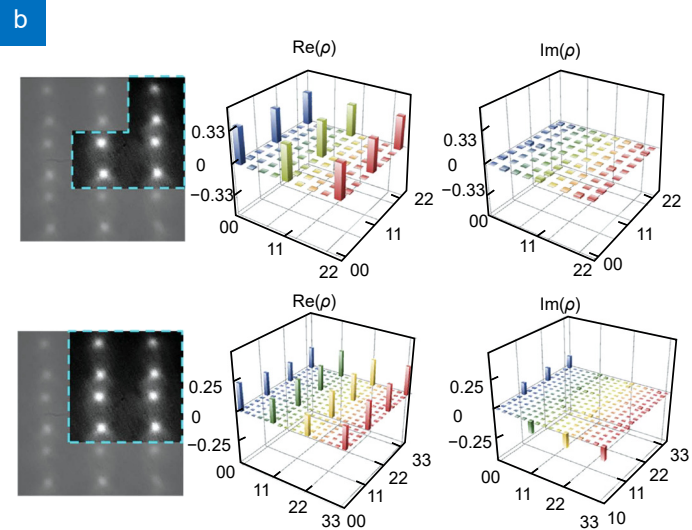

d
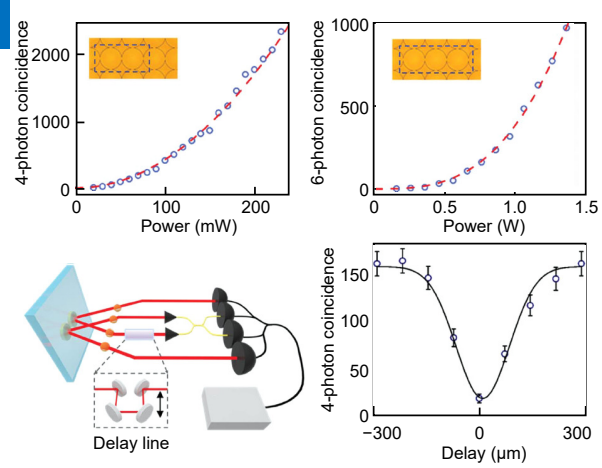

Fig. 1 | Quantum source based on metasurfaces. (a) Schematic of quantum source based on metasurfaces. (b) Characterization of three-dimensional, four-dimensional two-photon quantum states. (c) Manipulating the phase gradient of metasurface to prepare four kinds of bell states. (d) Characterization of multiphoton quantum source based on metasurface. Figure reproduced from: (a-d) ref. ${ }^{88}$, The American Association for the Advancement of Science.

as shown in Fig. 1(b), according to the quantum state tomography, the fidelities of three-dimensional and four-dimensional photon pair quantum state are $96.6 \%$ and $95 \%$ respectively. Moreover, manipulating the phase gradient of metasurface can control the generation of quantum state, and the phase difference between adjacent individual metalens element is $0, \pi / 2, \pi, 3 \pi / 2$ respectively, which leads to four bell states as follows:

$$
\begin{aligned}
& (|00\rangle+|11\rangle) / \sqrt{2}, \\
& (|00\rangle+\mathrm{i}|11\rangle) / \sqrt{2}, \\
& (|00\rangle-|11\rangle) / \sqrt{2}, \\
& (|00\rangle-\mathrm{i}|11\rangle) / \sqrt{2} .
\end{aligned}
$$

The density matrix reconstruction of four bell states is shown in Fig. 1(c) with high average fidelity of $97.9 \%$. This implies that the rich phase control associated with metasurface can be fully exploited to prepare desired quantum states. Morever, because the photon pair generated from metasurface is path entangled, this complex structure can easily realize compact, stable high dimensional multiphoton quantum source. As shown in Fig. $1(\mathrm{~d})$, the pump power dependencies of four-photon and six-photon source coincidence counts satisfy quadratic and cubic relationship respectively, which adequately confirms the preparation of four-photon and six-photon quantum source. Specifically, for two photon pairs independently generated from two different metalenses, one photon from each photon pair is used as the trigger, and another from each photon pair interfere at a $50: 50$ fiber beam splitter, resulting in $86.3 \%$ HOM dip visibility. Optical metasurface indeed has the capability to provide a stable, compact high dimensional multiphoton quantum source. However, preparing desirable quantum states through other degree of freedoms based on optical metasurface has not been displayed and may be hopefully achieved.

\section{Manipulation of quantum states}

In the quantum photonics regime, the manipulation of quantum states is indispensable to realizing quantum logic gates. Usually, this aim can be fulfilled by linear optical elements, such as wave plates, splitters and so on. As a consequence of extraordinary ability to control phase and amplitude of light, metasurface can realize almost all functionalities provided by linear optical elements in a 
smaller scale and multifunctional way, so the functionality associated with linear optical devices can be directly transplanted into optical metasurface with high integration density and efficiency.

Optical metasurface has two kinds of methods to manipulate the phase of light: resonant phase and geometrical phase. For the latter, the vortex beam can be generated through designing metasurface with azimuthal phase distribution. In the quantum physics regime, this space-variant spin-dependent phase changes orbital angular momentum of photons based on the corresponding spin angular momentum, and the difference of orbital angular momentum depends on the phase distribution of metasurface. As shown in Fig. 2(a), by utilizing the geometrical phase of metasurface, photon with different polarization acquires different orbital angular momentum, the process of photons through the metasurface is presented as
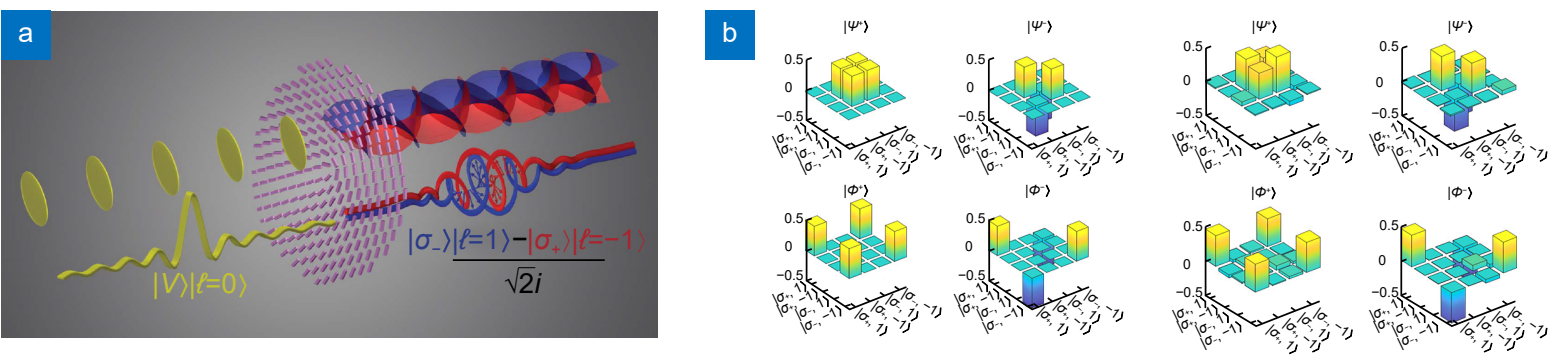
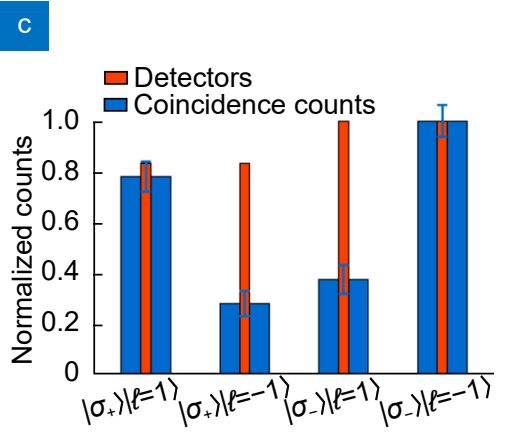

d

$\mathrm{N}$-photon polarization state

Density matrix

$\rho$

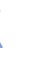

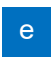
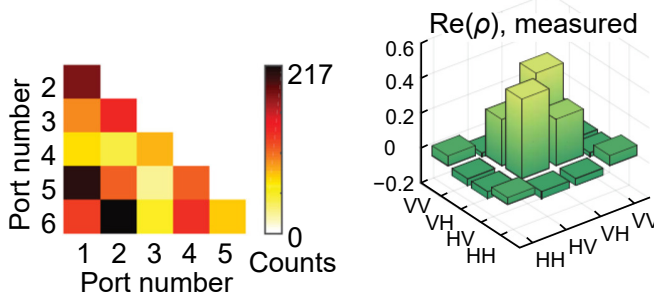

$\operatorname{Re}(\rho)$, measured
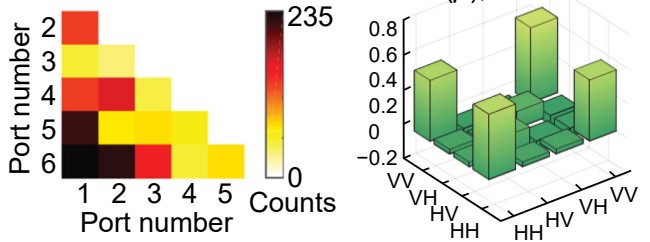

$\operatorname{Re}(\rho)$, predicted
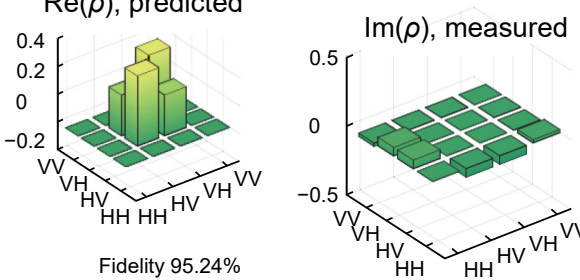

$\operatorname{Im}(\rho)$, predicted

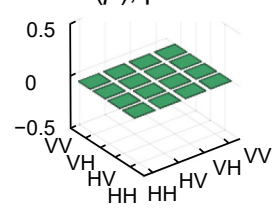

$\operatorname{Re}(\rho)$, predicted
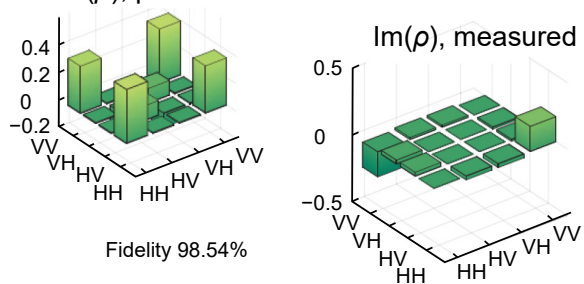

Fig. 2 | Manipulation of quantum states based on metasurface. (a) Employing geometric phase associated with metasurface to make photons with different spin polarization state acquire different orbital angular momentum. (b) Four bell states of single photon with entanglement of spin angular momentum and orbital angular momentum. (c) The mutual entanglement between a photon pair. (d) Left is the schematic of quantum state tomography based on metasurface, top right is three different inter-leaved metasurface, middle-right is the graphical representation of 6 different polarization states, bottom right is relationship between the minimum number of channels required and the number of photons. (e) The reconstruction of density matrix of two different photon pair state with fidelities 95.24\%, 98.54\% respectively. Figure reproduced from: (a-c) ref. ${ }^{89}$,The American Association for the Advancement of Science; (d-e) ref. ${ }^{90}$, The American Association for the Advancement of Science. 


$$
\left|\sigma_{ \pm}\right\rangle|l\rangle \rightarrow\left|\sigma_{\mp}\right\rangle|l \pm \Delta l\rangle
$$

$\sigma_{+}, \sigma_{-}, l, \Delta l$ represents right-handed rotation, lefthanded rotation, the orbital angular momentum a single photon carries and the difference of orbital angular momentum after single photon passes through metasurface, respectively ${ }^{89}$. The quantum state of a horizontally polarized single photon with zero orbital angular momentum is presented as

$$
|\boldsymbol{H}\rangle|l=0\rangle=\frac{1}{\sqrt{2}}\left(\left|\sigma_{+}\right\rangle+\left|\sigma_{-}\right\rangle\right)|l=0\rangle,
$$

where $\boldsymbol{H}$ represents the horizontal polarization. After the photon passes through metasurface, its quantum state is presented as

$$
\frac{1}{\sqrt{2}}\left(\left|\sigma_{+}\right\rangle|l=-\Delta l\rangle+\left|\sigma_{-}\right\rangle|l=\Delta l\rangle\right) .
$$

Subsequently, the entanglement between spin angular momentum and orbital angular momentum of single photon is realized. Designing metasurface with different phase distribution or flipping the metasurfacec can carry out different quantum operation on single photon. Figure 2(b) shows the four bell states of single photon with entanglement between spin angular momentum and orbital angular momentum. Based on the same principle, the entanglement of spin angular momentum of one photon with orbital angular momentum of another photon from the same photon pair is acquired, as shown in Fig. 2(c). When the spin polarization state of one photon is $\left|\sigma_{+}\right\rangle$, the probability of another photon having orbital angular momentum value of 1 is obviously higher than that of having orbital angular momentum value of -1 , and vice versa.

Moreover, owing to the extraordinary ability of beam splitting, optical metasurfaces can also be employed to carry out projective measurements on multiphoton quantum state ${ }^{90}$. As shown in Fig. 2(d), combining $M / 2$ kinds of metasurfaces to construct a new meta-gratings, among the interleaved metasurfaces, each one of them can split two different orthogonal polarization state of photons, which in total leads to $M$ kinds of polarization state. When $N$ photon quantum state interfere on the metasurface, the correlation among the behavior of photons that split into different channels exists. According to the measurement of different combinations of polarization channels, the density matrix of $N$ multiphoton state can be deduced by the coincidences of a complete set of measurements. Specifically, for the two photon state, three different metasurfaces are combined to ac- quire six beam splitting channels. The reconstruction of density matrix of two-dimensional biphoton state requires at least fifteen sets of different measurement bases, which can be realized by randomly selecting two of the six beam splitting channels. Figure 2(e) reconstructs the density matrix of two-dimensional biphoton states for two different quantum states, whose fidelities are $95.24 \%$ and $98.54 \%$ respectively. When the number of photons is high, the advantage of this method is more obvious because it can significantly simplify the experiment set-up and lower the requirement of number of detectors, greatly increasing the speed and stability of quantum state tomography.

Compared with all dielectric metasurface, although metallic metasurface brings inevitable loss that can reduce the coherence of light, this weakness can be transformed into advantages for particular application scenarios. For example, by designing metallic metasurface whose absorption is dependent on the polarization, the probability of specific polarization constitution of nonmaximal entangled state can be reduced, which then increases the entanglement of quantum state and realizes the distillation of quantum state ${ }^{91}$. Moreover, metallic metasurface have better thermal conductivity than dielectric metasurfaces. The temperature of metallic metasurfaces can be controlled through laser illumination, then the response of metasurface to light is modulated so as to actively control quantum state ${ }^{92}$.

\section{Quantum optical applications}

Before introducing applications of quantum optics based on metasurfaces, one important thing should be recalled is that the behavior of any type of light definitely can be described by quantum mechanics, but some quantum source can only be explained by quantum mechanics instead of classic electrodynamics, such as squeezed state, single or two photon state and so on. When the intensity of light is down to a few photons, probabilistic behavior of photon must be taken into consideration, and then many unapproachable applications in classical regime is able to be realized in quantum regime. In the classical photonic domain, the absorption of photons is probabilistic and controlled by the extinction spectrum of nanostructures. However, in the quantum regime, classical limit of absorption can be broken through combining the loss of metallic metasurfaces and quantum interference ${ }^{93,94,95}$. As shown in Fig. 3(a), the absorption of single photon in metallic metasurface is intrinsically 


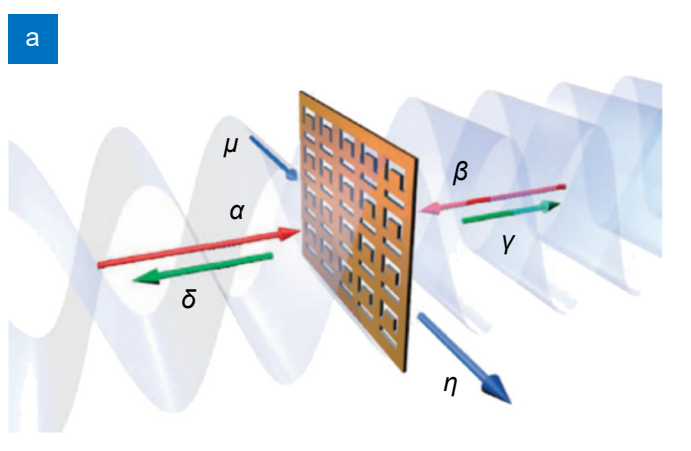

c

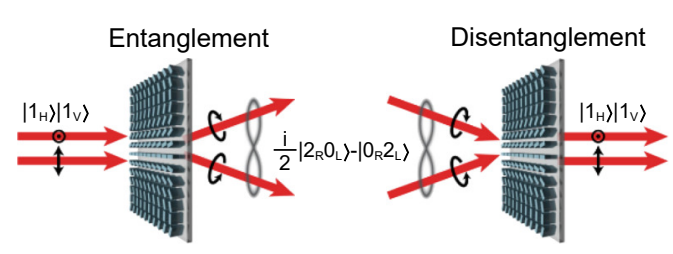

b
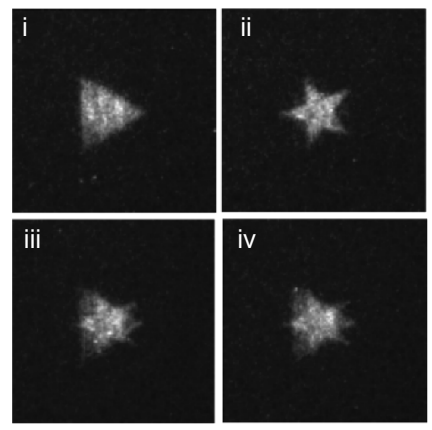

d
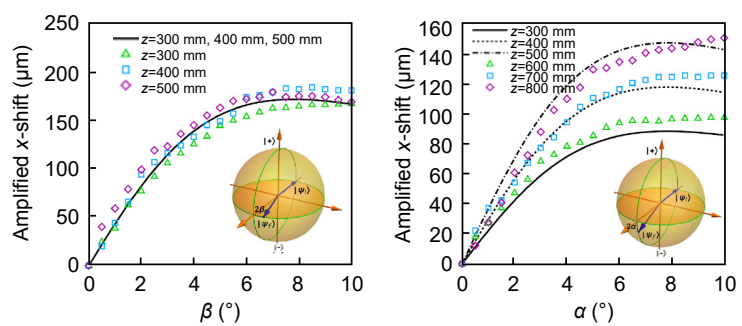

Fig. 3 | Quantum photonic applications based on metasurfaces. (a) Absorption of single photon based on metallic metasurfaces, $\alpha$ and $\beta$ represent two photon input channels, $\delta$ and $\gamma$ represent two photon output channels, $\mu$ and $\eta$ represent plasmonic input and output channel respectively. (b) Figure i and ii use signal photon of photon pair to image the metasurface, projective measurements on idle photon is carried out to clearly distinguish triangle and star pattern. Figure iii and iv use photons of mixed state to image metasurface which cannot distinguish the two patterns. (c) Metasurface is used to entangle and disentangle two photon NOON state. (d) The position shift and momentum shift of photon wavefunction induced by quantum weak measurement based on metasurfaces. Figure reproduced from: (a) ref. ${ }^{93}$, Springer Nature; (b) ref. ${ }^{96}$, American Physical Society; (c) ref. ${ }^{98}$, Springer Nature; (d) ref..$^{99}$, AIP Publishing.

probabilistic if single photon impinges on the metasurface from only one side. Things change when single photon arrives on the metasurface simultaneously from both sides, and this can be realized through employing the superposition state encoded in path. Because of quantum interference between the two paths, single photon can be deterministically absorbed beyond the classical limit of probability $50 \%{ }^{93}$. Moreover, through utilizing the nonlocal correlation between photons from photon pair, the projective measurement of quantum state of idle photon can manipulate the absorption response of metasurface to the signal photon ${ }^{94}$. In this way whether signal photon is absorbed can be deterministically controlled. Furthermore, metasurface can also enhance absorption of multiphoton states ${ }^{95}$. In general, multiphoton process is weak and has nonlinear dependence on the amplitude of incident light, which severely limits the absorption of multiphoton at the few photons level. Metasurface placed inside a Sagnac style interferometer is able to absorb two photon NOON state with efficiency up to $40 \%$, which is much as twice as that of linear absorption process.

Metasurface can also be employed in quantum ima- ging $^{96}$. As shown in Fig. 3(b), two overlaid pattern is simultaneously fabricated on the same metasurface: triangle and star. Different pattern can only allow specific polarization state light pass through. When signal photon from photon pair is used to image the metasurface, selective measurement of idle photon can determine which pattern can be distinguished. And as the entanglement of photon pair decreases, the discrimination of the two patterns decreases. Based on the same principle, nonlocal effects of entangled photon pair can be used to remotely control quantum edge detection. Pancharatnam-Berry phase can convert one image into two overlapped image when light passes through metasurfaces, and the polarization state in the region of edge and non-edge is different. Therefore, with the help of post-selection, which only detects the horizontal polarization state, quantum edge detection switch is realized ${ }^{97}$.

In the meantime, there are many application scenarios for optical metasurfaces in quantum measurement ${ }^{98,99}$. As shown in Fig. 3(c), the quantum state of entangled photon pair with orthogonal linear polarization is presented as $\left|1_{\mathrm{H}}\right\rangle\left|1_{\mathrm{V}}\right\rangle$. When it is represented based on circular polarization bases, its quantum state is 
presented as

$$
-\frac{\mathrm{i}}{2}\left(\left|2_{\mathrm{L}}\right\rangle|0\rangle-|0\rangle\left|2_{\mathrm{R}}\right\rangle\right)
$$

which is a two photon NOON state. After the photon pair passes through metasurface, the left, right handed polarization photon irradiates into different direction as a consequence of geometrical phase of metasurface. Then photon pair is spatially entangled and disentangled dependent on the times of photon pair passing through metasurface. Based on this principle, P. Georgi et al. constructed an interferometer consisting of metasurface and other optical elements ${ }^{98}$. Tilting a $130-\mu \mathrm{m}$-thick glass plate in one of the two paths can change the phase difference between the two optical paths. It has been demonstrated that the count rates at single detector does not change with the introduced phase, but the coincidence counts oscillate with the variation of phase difference, this adequately confirmed that the entangled photon pair still kept good coherence after passing through metasurface. When the time delay between the two input photons increases, the visibility of interference fringes from the MBI decreases, so does the visibility of the coincidence rates. Quantum HOM interference visibility of $86.8 \pm 1.1 \%$ can be obtained for two photons illuminating on metasurface simultaneously. Moreover, not only can metasurface be used to carry out projective measurement, it can also be utilized to carry out quantum weak measurement. Quantum weak measurement that firstly derived from electronic system is an important branch of quantum measurement. By applying weak magnetic field on the electron, the quantum weak measurement of electron spin quantum state can be made. The main principle of quantum weak measurement is to extract the information about quantum state without disturbing the quantum state, and this method has been applied to wavefunction measurement and weak signal amplification $^{100,101}$. In analogy with electronic system, weak phase gradient on metasurface in photonic system corresponds to the weak magnetic field in the electronic system. By designing the weak gradient on the metasurface, the quantum weak measurement of photon quantum state can be made ${ }^{99}$. In the experiment apparatus of quantum weak measurement, GLP1 (Glan Laser polarizer) is used to prepare the initial state, metasurface provides weak phase gradient, and quarter wave plate and GLP2 are used for post-selection by selecting different final state, changing the angle $\beta$ between the fast axis of quarter wave plate and $y$ axis as well as the angle $\alpha+\beta$ between the GLP2 and the $x$ axis. As shown in Fig. 3(d), when $\alpha=0$ is satisfied, weak value is purely imaginary. As the angle $\beta$ increases, the shift of photon spot increases. But when the position of CCD gets further, there is no change in the shift of photon spot. This confirms that purely imaginary weak value corresponds to the position shift of photon wavefunction. When $\beta=0$ is satisfied, weak value is purely real. As the angle $\alpha$ increases, the shift of photon spot increases. And when the position of CCD gets further, the shift of photon spot gets larger, which confirms that purely real weak value corresponds to the momentum shift of photon wavefunction.

\section{Quantum vacuum engineering of quantum emitter}

Initially, the lifetime of quantum emitter was thought to be intrinsic and independent of the external electromagnetic field environment until Purcell proposed that the lifetime of quantum emitter can be manipulated, e.g. the radiative rate and non-radiative rate of quantum emitters can be suppressed or enhanced by changing the electromagnetic field environment surrounding the quantum emitters. Then, the researchers began to design nanoscale optical structures with small mode volume or high $Q$ value to enhance the interaction between quantum emitters and optical structures in space and time dimension. In the weak coupling regime, the fluorescence lifetime of quantum emitter is modulated. In the strong coupling regime, the energy levels of quantum emitters and optical structures are hybridized. Rabi splitting can be observed in absorption spectrum, scattering spectrum or fluorescence spectrum.

All of these works control the radiation behavior of quantum emitter in the near field of optical structure. The precise control of the position of quantum emitter and high loss near metallic nanostructure limits their development. In order to solve these problems, it is necessary to modulate the radiation behavior of quantum emitter in the far field of optical structure. Metasurface can achieve this goal due to its excellent phase control ability. P. K. Jha et al. systematically studied the quantum vacuum engineering of quantum emitter through metasurface $^{102-104}$. As shown in Fig. 4(a), the metasurface breaks the quantum vacuum symmetry of quantum emitter, causing quantum interference of multiple energy levels of quantum emitter. This effect is forbidden in free space ${ }^{102}$. Specifically, as shown in Fig. 4(b), through judiciously designing metasurface, it is made to 


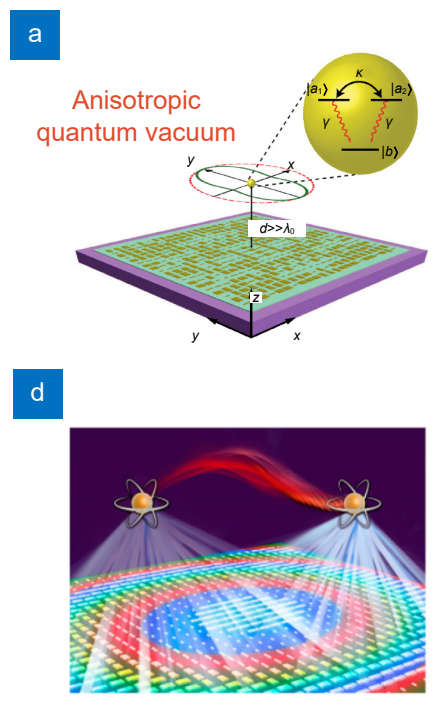

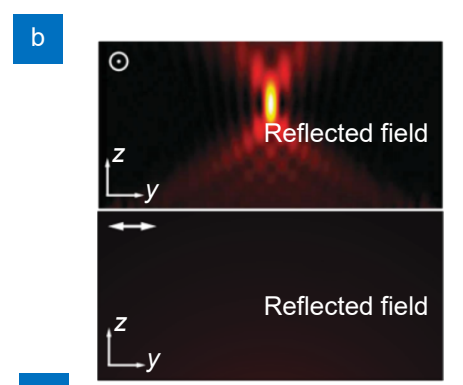

e

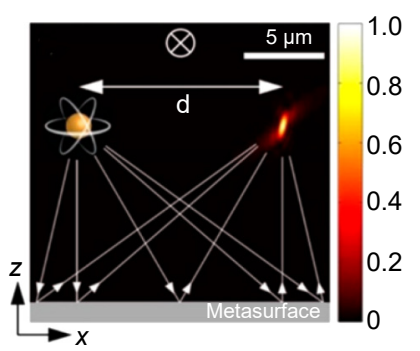

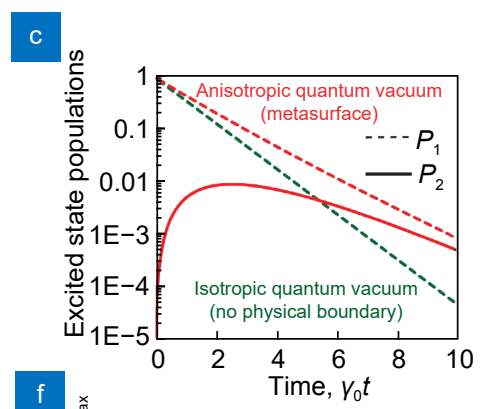

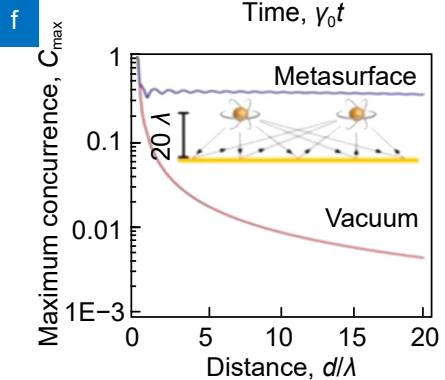

Fig. 4 | Quantum vacuum engineering based on metasurfaces. (a) Based on metasurfaces, the quantum vacuum symmetry of quantum emitter is broken, so that quantum interference between different energy levels of multi-level quantum emitter occurs. (b) The electromagnetic field radiated by an electric dipole in the $x$ direction above metasurface can be focused back to the source point along the original path with the maximum efficiency being $81 \%$ and the electromagnetic field radiated by an electric dipole in the $y$ direction has no such effect. (c) When there is no metasurface, the energy level $\left|a_{1}\right\rangle$ decayed exponentially and the energy level $\left|a_{2}\right\rangle$ occupied 0 . When the metasurface exists, the decay rate of the energy level $\left|a_{1}\right\rangle$ decreases, and the energy level $\left|a_{2}\right\rangle$ population firstly increases and then decreases. (d) Schematic diagram of quantum entanglement of two quantum emitters based on metasurface. (e) The metasurface enables the electromagnetic field radiated by the source point electric dipole to be oriented to the position of the target electric dipole with highest efficiency $82 \%$. (f) The concurrence of two quantum emitters varies with the distance between them, the red solid line corresponds to the vacuum condition, and the blue solid line corresponds to the metasurface condition. Figure reproduced from: (a-c) ref. ${ }^{102}$, American Physical Society; (d-e) ref. ${ }^{103}$, American Physical Society.

have the polarization dependent response. Namely when the electric dipole is along the $x$ direction, its radiation field returns back to the source point along the original way with the maximum efficiency $81 \%$. When the electric dipole is along the $y$ direction, there is no such effect. From the perspective of quantum mechanics, the metasurface with such function breaks the quantum vacuum symmetry of quantum emitter. The theoretical results are shown in Fig. 4(c). When there is no metasurface, the energy level $\left|a_{1}\right\rangle$ decayed exponentially and the energy level $\left|a_{2}\right\rangle$ occupied 0 . When the metasurface exists, the decay rate of the energy level $\left|a_{1}\right\rangle$ decreases, and the energy level $\left|a_{2}\right\rangle$ population firstly increases and then decreases. This shows that the introduction of anisotropic quantum vacuum through metasurface leads to the population transfer from energy level $\left|a_{1}\right\rangle$ to energy level $\left|a_{2}\right\rangle$ because of the existence of optical metasurface which releases the stringent requirement of nonorthogonal transition.

Furthermore, when two quantum emitters are considered, as shown in Fig. 4(d) and 4(e). The metasurface enables the electromagnetic field radiated by the electric dipole of source point to focus on the position of the target electric dipole in a directional manner with the highest efficiency being $82 \%$, which leads to entanglement between the two quantum emitters ${ }^{103}$. As shown in Fig. 4(f). When the metasurface exists, concurrence of the two quantum emitters is enhanced by two orders of magnitude than that of without the metasurface. Concurrence is the figure of merit to measure the degree of entanglement of two quantum emitters. As the distance between the two quantum emitters increases, concurrence falls rapidly in the condition of no metasurface. When the metasurface exists, concurrence falls more slowly and remains great in a long distance. Based on the same principle, the quantum vacuum of transition metal dichalcogenides monolayers can be engineered by geometrical phase of metasurface, which realizes spontaneous generation of exciton intervalley coherence ${ }^{104}$. In free space, the energy-degenerate excitons at the points $\mathrm{K}$ and $\mathrm{K}$ ' of the Brillouin zone of transition metal dichalcogenides monolayers can only be excited by left-handed and right-handed polarized light respectively, and the fluorescence corresponds to left-handed and righthanded polarized light respectively. By using metasurface, the left-handed polarized light of the valley exciton radiation at the source point can be focused back to the 
source point with the highest efficiency $47 \%$. Since lefthanded polarized light becomes right-handed polarized light after reflection, the $\mathrm{K}$ valley exciton can indirectly excite the $K^{\prime}$ valley exciton, and vice versa.

Moreover, when metasurface is fully anisotropic, adjusting quantization axis of quantum emitter leads to an observable difference both in temporal dynamics and spectral properties of the quantum emitters which are initially pumped into states of opposite helicities, which confirms that optical activity can originate from the quantum interference of the multiple decay channels of quantum emitter ${ }^{105}$. In addition, the energy level structure of quantum emitter is of great significance for the experimental realization of quantum vacuum manipulation. Because type $V$ quantum emitter has two excited states and one ground state, type $\Lambda$ quantum emitter has one excited state and two ground states. Compared with type $\mathrm{V}$ quantum emitter, type $\Lambda$ quantum emitter has longer coherence time between energy levels ${ }^{106}$.

\section{Conclusion and outlook}

In this paper, we have summarized recent works related to quantum optics based on metasuraces. We firstly introduce some researches related to quantum plasmonics. Despite of the intrinsic loss and decoherence associated with metal nanostructure, novel quantum phenomenon, such as quantum entanglement, quantum tunneling and quantum self-interference have been demonstrated. We then review quantum source based on metasurface, high dimensional multiphoton quantum source encoded in path degree of freedom is realized. Obviously it's just not enough to generate path-entangled photon pairs, and we need to distribute quantum states over a long distance and realize practical application of information processing. Current preferred method is via the multi-core fiber, but it's hard to stabilize the phase difference between different channels. Alternatively, mode sorter as quantum interface to convert the path entanglement to angular orbital momentum (OAM) entanglement has been shown. However, light carried with OAM still can't propagate in free space or fiber links over a long distance, hence, it's necessary to convert the information encoded in path or OAM into other degrees of freedom which is more robust and stable against the environment noise. We then discuss recent techniques about manipulation of quantum states based on metasurfaces. By judiciously designing metasurfaces, the functionality of beam splitting has been utilized to enhance the speed and stability of quantum state tomography, but how to design metasurfaces to combine multiple incoherent output beams into one is still challenging and illusive. Achieving this goal can circumvent the complexities of high dimensional quantum state tomography. Moreover, we have shown that metasuface can also provide unique opportunities for quantum imaging, quantum sensing, quantum measurement and some other applications. Interestingly, although the intrinsic loss of metallic metasurfaces is detrimental to the coherence of photons, this weakness can be utilized to enhance and control the absorption of photon. Finally, we review related works about the interaction between metasurfaces and quantum emitters. The radiation behavior can be manipulated by changing the quantum vacuum surrounding the quantum emitter. Nowadays single photon source is mostly based on quantum dots as a consequence of its deterministic generation. But it still remains unknown whether single photon pair can be highly efficiently generated through the combination of metasurface and quantum emitter.

Compared with other linear optical elements, metasuface possesses richer ability to control the phase of light and smaller scale which render metasurface suitable for highly integrated quantum optical systems. The method to directly map the abundant light field manipulation functionality in the classical domain to quantum domain through metasurface is a general way to find optical metasurface's position in quantum optics. Despite the great advances that has been achieved, the potential of metasurfaces for quantum optics has not yet been fully exploited, and some problems and challenges remain to be solved. For example, the phase encoding potential embedded in metasurface may be applied to super dense coding and quantum communication. As for manipulation of quantum states, preparing desired quantum states through electric control of metasurface is appealing and has great influence on the development of quantum communication and information. Apart from this, the most important is to know unknown, and it may be an arduous task to discover new physics through traditional optical elements. Optical metasurfaces can make experiment and measurement simple and convenient, providing a brand new and very promising platform to study quantum optics.

\section{References}

1. Shalaev VM. Optical negative-index metamaterials. Nat 
Photonics 1, 41-48 (2007).

2. Valentine J, Zhang S, Zentgraf T, Ulin-Avila E, Genov DA et al. Three-dimensional optical metamaterial with a negative refractive index. Nature 455, 376-379 (2008).

3. Moitra P, Yang YM, Anderson Z, Kravchenko II, Briggs DP et al. Realization of an all-dielectric zero-index optical metamaterial. Nat Photonics 7, 791-795 (2013).

4. Fang $N$, Zhang $X$. Imaging properties of a metamaterial superlens. In Proceedings of the 2nd IEEE Conference on Nanotechnology 225-228 (IEEE, 2002); http://doi.org/10.1109/NANO.2002.1032233

5. Jacob Z, Alekseyev LV, Narimanov E. Optical hyperlens: farfield imaging beyond the diffraction limit. Opt Express 14, 8247-8256 (2006).

6. Liu ZW, Lee H, Xiong Y, Sun C, Zhang X. Far-field optical hyperlens magnifying sub-diffraction-limited objects. Science 315, 1686 (2007).

7. Cai WS, Chettiar UK, Kildishev AV, Shalaev VM. Optical cloaking with metamaterials. Nat Photonics 1, 224-227 (2007).

8. Smolyaninov II, Smolyaninova VN, Kildishev AV, Shalaev VM. Anisotropic metamaterials emulated by tapered waveguides: application to optical cloaking. Phys Rev Lett 102, 213901 (2009).

9. Yu NF, Genevet P, Kats MA, Aieta F, Tetienne JP et al. Light propagation with phase discontinuities: generalized laws of reflection and refraction. Science 334, 333-337 (2011).

10. Klein MW, Enkrich C, Wegener M, Linden S. Second-harmonic generation from magnetic metamaterials. Science $\mathbf{3 1 3}$, 502-504 (2006).

11. Kim S, Jin J, Kim YJ, Park IY, Kim Y et al. High-harmonic generation by resonant plasmon field enhancement. Nature $\mathbf{4 5 3}$, 757-760 (2008).

12. Lee J, Tymchenko M, Argyropoulos C, Chen PY, Lu F et al. Giant nonlinear response from plasmonic metasurfaces coupled to intersubband transitions. Nature 511, 65-69 (2014).

13. Luu TT, Garg M, Kruchinin SY, Moulet A, Hassan MT et al. Extreme ultraviolet high-harmonic spectroscopy of solids. Nature 521, 498-502 (2015).

14. Kuznetsov Al, Miroshnichenko AE, Brongersma ML, Kivshar YS, Luk'yanchuk B. Optically resonant dielectric nanostructures. Science 354, aag2472 (2016).

15. Sivis M, Taucer M, Vampa G, Johnston K, Staudte A et al. Tailored semiconductors for high-harmonic optoelectronics. Science 357, 303-306 (2017).

16. Koshelev K, Kruk S, Melik-Gaykazyan E, Choi JH, Bogdanov $A$ et al. Subwavelength dielectric resonators for nonlinear nanophotonics. Science 367, 288-292 (2020).

17. Bomzon ZE, Biener G, Kleiner V, Hasman E. Space-variant Pancharatnam-Berry phase optical elements with computergenerated subwavelength gratings. Opt Lett 27, 1141-1143 (2002).

18. Zayats AV, Smolyaninov II, Maradudin AA. Nano-optics of surface plasmon polaritons. Phys Rep 408, 131-314 (2005).

19. Bouhelier A, Beversluis M, Hartschuh A, Novotny L. Near-field second-harmonic generation induced by local field enhancement. Phys Rev Lett 90, 013903 (2003).

20. Kim EM, Elovikov SS, Murzina TV, Nikulin AA, Aktsipetrov OA et al. Surface-enhanced optical third-harmonic generation in Ag island films. Phys Rev Lett 95, 227402 (2005).

21. Kinkhabwala A, Yu ZF, Fan SH, Avlasevich $Y$, Müllen $K$ et al.
Large single-molecule fluorescence enhancements produced by a bowtie nanoantenna. Nat Photonics 3, 654-657 (2009).

22. Renger J, Quidant R, van Hulst N, Novotny L. Surface-enhanced nonlinear four-wave mixing. Phys Rev Lett 104, 046803 (2010).

23. Park IY, Kim S, Choi J, Lee DH, Kim YJ et al. Plasmonic generation of ultrashort extreme-ultraviolet light pulses. Nat Photonics 5, 677-681 (2011).

24. Sivis M, Duwe M, Abel B, Ropers C. Extreme-ultraviolet light generation in plasmonic nanostructures. Nat Phys 9, 304-309 (2013).

25. Aouani H, Rahmani M, Navarro-Cia M, Maier SA. Third-harmonic-upconversion enhancement from a single semiconductor nanoparticle coupled to a plasmonic antenna. Nat Nanotechnol 9, 290-294 (2014).

26. Zhu WQ, Crozier KB. Quantum mechanical limit to plasmonic enhancement as observed by surface-enhanced Raman scattering. Nat Commun 5, 5228 (2014).

27. Han S, Kim H, Kim YW, Kim YJ, Kim S et al. High-harmonic generation by field enhanced femtosecond pulses in metalsapphire nanostructure. Nat Commun 7, 13105 (2016).

28. Zhong JH, Vogelsang J, Yi JM, Wang D, Wittenbecher $L$ et al. Nonlinear plasmon-exciton coupling enhances sum-frequency generation from a hybrid metal/semiconductor nanostructure. Nat Commun 11, 1464 (2020).

29. Zhang XY, Cao QT, Wang Z, Liu YX, Qiu CW et al. Symmetrybreaking-induced nonlinear optics at a microcavity surface. Nat Photonics 13, 21-24 (2019).

30. Miroshnichenko AE, Evlyukhin AB, Yu YF, Bakker RM, Chipouline $A$ et al. Nonradiating anapole modes in dielectric nanoparticles. Nat Commun 6, 8069 (2015).

31. Grinblat G, Li Y, Nielsen MP, Oulton RF, Maier SA. Enhanced third harmonic generation in single germanium nanodisks excited at the anapole mode. Nano Lett 16, 4635-4640 (2016).

32. Carletti L, Koshelev K, De Angelis C, Kivshar Y. Giant nonlinear response at the nanoscale driven by bound states in the continuum. Phys Rev Lett 121, 033903 (2018).

33. Liu ZJ, $X u Y$, Lin $Y$, Xiang J, Feng TH et al. High-Q quasibound states in the continuum for nonlinear metasurfaces. Phys Rev Lett 123, 253901 (2019).

34. Yang YM, Wang WY, Boulesbaa A, Kravchenko II, Briggs DP et al. Nonlinear fano-resonant dielectric metasurfaces. Nano Lett 15, 7388-7393 (2015).

35. Carletti L, Locatelli A, Stepanenko O, Leo G, De Angelis C. Enhanced second-harmonic generation from magnetic resonance in AlGaAs nanoantennas. Opt Express 23, 26544-26550 (2015).

36. Shcherbakov MR, Neshev DN, Hopkins B, Shorokhov AS, Staude I et al. Enhanced third-harmonic generation in silicon nanoparticles driven by magnetic response. Nano Lett 14, 6488-6492 (2014).

37. Kruk S, Poddubny A, Smirnova D, Wang L, Slobozhanyuk A et al. Nonlinear light generation in topological nanostructures. Nat Nanotechnol 14, 126-130 (2019).

38. Smirnova D, Kruk S, Leykam D, Melik-Gaykazyan E, Choi DY et al. Third-harmonic generation in photonic topological metasurfaces. Phys Rev Lett 123, 103901 (2019).

39. Wang SM, Wu PC, Su VC, Lai YC, Chu CH et al. Broadband achromatic optical metasurface devices. Nat Commun 8, 187 (2017). 
40. Wang SM, Wu PC, Su VC, Lai YC, Chen MK et al. A broadband achromatic metalens in the visible. Nat Nanotechnol 13, 227-232 (2018).

41. Ye WM, Zeuner F, Li X, Reineke B, He S et al. Spin and wavelength multiplexed nonlinear metasurface holography. Nat Commun 7, 11930 (2016).

42. Ni XJ, Kildishev AV, Shalaev VM. Metasurface holograms for visible light. Nat Commun 4, 2807 (2013).

43. Zheng GX, Mühlenbernd H, Kenney M, Li GX, Zentgraf T et al. Metasurface holograms reaching $80 \%$ efficiency. Nat Nanotechnol 10, 308-312 (2015).

44. Huang LL, Chen XZ, Mühlenbernd H, Zhang H, Chen SM et al. Three-dimensional optical holography using a plasmonic metasurface. Nat Commun 4, 2808 (2013).

45. Yin XB, Ye ZL, Rho J, Wang Y, Zhang X. Photonic spin Hall effect at metasurfaces. Science 339, 1405-1407 (2013).

46. Yang YM, Wang WY, Moitra P, Kravchenko II, Briggs DP et al. Dielectric meta-reflectarray for broadband linear polarization conversion and optical vortex generation. Nano Lett 14, 1394-1399 (2014)

47. Karimi E, Schulz SA, De Leon I, Qassim H, Upham J et al. Generating optical orbital angular momentum at visible wavelengths using a plasmonic metasurface. Light Sci App/ 3, e167 (2014).

48. Cerf NJ, Bourennane M, Karlsson A, Gisin N. Security of quantum key distribution using d-level systems. Phys Rev Lett 88, 127902 (2002).

49. Gisin N, Ribordy GG, Tittel W, Zbinden H. Quantum cryptography. Rev Mod Phys 74, 145-195 (2002).

50. Gisin N, Thew R. Quantum communication. Nat Photonics 1, 165-171 (2007)

51. Scarani $V$, Bechmann-Pasquinucci $H$, Cerf $N J$, Dušek $M$, Lütkenhaus $\mathrm{N}$ et al. The security of practical quantum key distribution. Rev Mod Phys 81, 1301-1350 (2009).

52. Lo HK, Curty M, Tamaki K. Secure quantum key distribution. Nat Photonics 8, 595-604 (2014).

53. Wang XL, Cai XD, Su ZE, Chen MC, Wu D et al. Quantum teleportation of multiple degrees of freedom of a single photon. Nature 518, 516-519 (2015)

54. Knill E, Laflamme R, Milburn GJ. A scheme for efficient quantum computation with linear optics. Nature 409, 46-52 (2001).

55. O'Brien JL. Optical quantum computing. Science $\mathbf{3 1 8}$, 1567-1570 (2007).

56. Lanyon BP, Barbieri M, Almeida MP, Jennewein T, Ralph TC et al. Simplifying quantum logic using higher-dimensional Hilbert spaces. Nat Phys 5, 134-140 (2009).

57. Neeley M, Ansmann M, Bialczak RC, Hofheinz M, Lucero E et al. Emulation of a quantum spin with a superconducting phase qudit. Science 325, 722-725 (2009).

58. Kaltenbaek R, Lavoie J, Zeng B, Bartlett SD, Resch KJ. Optical one-way quantum computing with a simulated valence-bond solid. Nat Phys 6, 850-854 (2010).

59. Aspuru-Guzik A, Walther P. Photonic quantum simulators. Nat Phys 8, 285-291 (2012).

60. Georgescu IM, Ashhab S, Nori F. Quantum simulation. Rev Mod Phys 86, 153-185 (2014).

61. Giovannetti V, Lloyd S, Maccone L. Advances in quantum metrology. Nat Photonics 5, 222-229 (2011).

62. Pirandola S, Bardhan BR, Gehring T, Weedbrook C, Lloyd S.
Advances in photonic quantum sensing. Nat Photonics 12, 724-733 (2018).

63. You JQ, Nori F. Atomic physics and quantum optics using superconducting circuits. Nature 474, 589-597 (2011).

64. Diehl S, Micheli A, Kantian A, Kraus B, Büchler $\mathrm{H}$ et al. Quantum states and phases in driven open quantum systems with cold atoms. Nat Phys 4, 878-883 (2008).

65. Leibfried D, Blatt R, Monroe C, Wineland D. Quantum dynamics of single trapped ions. Rev Mod Phys 75, 281-324 (2003).

66. Li CL, Yu P, Huang YJ, Zhou Q, Wu J et al. Dielectric metasurfaces: from wavefront shaping to quantum platforms. Prog Surf Sci 95, 100584 (2020).

67. Uriri S, Ismail Y, Petruccione F. Quantum metamaterials: applications in quantum information science. arXiv: 2006.03757 (2020).

68. Rivera N, Kaminer I. Light-matter interactions with photonic quasiparticles. Nat Rev Phys 2, 538-561 (2020).

69. Solntsev AS, Agarwal GS, Kivshar YS. Metasurfaces for quantum photonics. arXiv: 2007.14722 (2020).

70. Heeres RW, Kouwenhoven LP, Zwiller V. Quantum interference in plasmonic circuits. Nat Nanotechnol 8, 719-722 (2013).

71. Fakonas JS, Lee H, Kelaita YA, Atwater HA. Two-plasmon quantum interference. Nat Photonics 8, 317-320 (2014).

72. Altewischer E, van Exter MP, Woerdman JP. Plasmon-assisted transmission of entangled photons. Nature $\mathbf{4 1 8}$, 304-306 (2002).

73. Moreno E, García-Vidal FJ, Erni D, Cirac JI, Martín-Moreno L. Theory of plasmon-assisted transmission of entangled photons. Phys Rev Lett 92, 236801 (2004).

74. Fasel S, Robin F, Moreno E, Erni D, Gisin N et al. Energy-time entanglement preservation in plasmon-assisted light transmission. Phys Rev Lett 94, 110501 (2005).

75. Huck A, Smolka S, Lodahl P, Sørensen AS, Boltasseva A et al. Demonstration of quadrature-squeezed surface plasmons in a gold waveguide. Phys Rev Lett 102, 246802 (2009).

76. Tan SF, Wu L, Yang JKW, Bai P, Bosman M et al. Quantum plasmon resonances controlled by molecular tunnel junctions. Science 343, 1496-1499 (2014).

77. Kolesov R, Grotz B, Balasubramanian G, Stöhr RJ, Nicolet AAL et al. Wave-particle duality of single surface plasmon polaritons. Nat Phys 5, 470-474 (2009).

78. Dheur MC, Devaux E, Ebbesen TW, Baron A, Rodier JC et al. Single-plasmon interferences. Sci Adv 2, e1501574 (2016).

79. Erhard M, Krenn M, Zeilinger A. Advances in high-dimensional quantum entanglement. Nat Rev Phys 2, 365-381 (2020).

80. Reck M, Zeilinger A, Bernstein HJ, Bertani P. Experimental realization of any discrete unitary operator. Phys Rev Lett 73, 58-61 (1994).

81. Krenn M, Hochrainer A, Lahiri M, Zeilinger A. Entanglement by path identity. Phys Rev Lett 118, 080401 (2017).

82. Wang JW, Paesani S, Ding YH, Santagati R, Skrzypczyk P et al. Multidimensional quantum entanglement with large-scale integrated optics. Science 360, 285-291 (2018).

83. Siomau M, Kamli AA, Moiseev SA, Sanders BC. Entanglement creation with negative index metamaterials. Phys Rev A 85, 050303 (2012).

84. Poddubny AN, lorsh IV, Sukhorukov AA. Generation of photon-plasmon quantum states in nonlinear hyperbolic metamaterials. Phys Rev Lett 117, 123901 (2016). 
85. Marino G, Solntsev AS, Xu L, Gili VF, Carletti L et al. Spontaneous photon-pair generation from a dielectric nanoantenna. Optica 6, 1416-1422 (2019).

86. Santiago-Cruz T, Sultanov V, Zhang HZ, Krivitsky LA, Chekhova MV. Spontaneous parametric down-conversion from subwavelength nonlinear films. arXiv: 2009.00324 (2020).

87. Ming Y, Zhang W, Tang J, Liu Y, Xia ZL et al. Photonic entanglement based on nonlinear metamaterials. Laser Photonics Rev 14, 1900146 (2020).

88. Li L, Liu ZX, Ren XF, Wang SM, Su VC et al. Metalens-arraybased high-dimensional and multiphoton quantum source. Science 368, 1487-1490 (2020).

89. Stav T, Faerman A, Maguid E, Oren D, Kleiner $\mathrm{V}$ et al. Quantum entanglement of the spin and orbital angular momentum of photons using metamaterials. Science $\mathbf{3 6 1}$, 1101-1104 (2018).

90. Wang K, Titchener JG, Kruk SS, Xu L, Chung HP et al. Quantum metasurface for multiphoton interference and state reconstruction. Science 361, 1104-1108 (2018).

91. Asano M, Bechu M, Tame M, Özdemir Ş K, Ikuta R et al. Distillation of photon entanglement using a plasmonic metamaterial. Sci Rep 5, 18313 (2015).

92. Uriri SA, Tashima T, Zhang X, Asano M, Bechu M et al. Active control of a plasmonic metamaterial for quantum state engineering. Phys Rev A 97, 053810 (2018).

93. Roger T, Vezzoli S, Bolduc E, Valente J, Heitz JJF et al. Coherent perfect absorption in deeply subwavelength films in the single-photon regime. Nat Commun 6, 7031 (2015).

94. Altuzarra C, Vezzoli S, Valente J, Gao WB, Soci C et al. Coherent perfect absorption in metamaterials with entangled photons. ACS Photonics 4, 2124-2128 (2017).

95. Lyons A, Oren D, Roger T, Savinov V, Valente J et al. Coherent metamaterial absorption of two-photon states with $40 \%$ efficiency. Phys Rev A 99, 011801 (2019).

96. Altuzarra C, Lyons A, Yuan GH, Simpson C, Roger T et al. Imaging of polarization-sensitive metasurfaces with quantum entanglement. Phys Rev A 99, 020101 (2019).

97. Zhou JX, Liu SK, Qian HL, Li YH, Luo HL et al. Metasurface enabled quantum edge detection. Sci Adv 6, eabc4385 (2020).

98. Georgi P, Massaro M, Luo KH, Sain B, Montaut $\mathrm{N}$ et al. Metasurface interferometry toward quantum sensors. Light Sci Appl 8, 70 (2019).

99. Chen SZ, Zhou XX, Mi CQ, Liu ZX, Luo $\mathrm{HL}$ et al. Dielectric metasurfaces for quantum weak measurements. Appl Phys Lett 110, 161115 (2017).

100. Lundeen JS, Sutherland B, Patel A, Stewart C, Bamber C. Direct measurement of the quantum wavefunction. Nature $\mathbf{4 7 4}$ 188-191 (2011).

101. Hosten $O$, Kwiat P. Observation of the spin hall effect of light via weak measurements. Science 319, 787-790 (2008).

102. Jha PK, Ni XJ, Wu C, Wang Y, Zhang X. Metasurface-enabled remote quantum interference. Phys Rev Lett 115 , 025501 (2015).

103. Jha PK, Shitrit N, Kim J, Ren XX, Wang $Y$ et al. Metasurfacemediated quantum entanglement. ACS Photonics 5, 971-976 (2018).

104. Jha PK, Shitrit N, Ren XX, Wang Y, Zhang X. Spontaneous exciton valley coherence in transition metal dichalcogenide monolayers interfaced with an anisotropic metasurface. Phys Rev Lett 121, 116102 (2018).

105. Kornovan D, Petrov M, lorsh I. Noninverse dynamics of a quantum emitter coupled to a fully anisotropic environment. Phys Rev A 100, 033840 (2019).

106. Lassalle E, Lalanne P, Aljunid S, Genevet P, Stout B et al. Long-lifetime coherence in a quantum emitter induced by a metasurface. Phys Rev A 101, 013837 (2020).

\section{Acknowledgements}

The authors are grateful that this work was supported by the National Key R\&D Program of China (2017YFA0303700, 2017YFA0303702, and 2016YFA0202103), the National Natural Science Foundation of China (No. 11822406, 11834007, 11774162, 11674166, 11674167, 11674168, 11621091, 11774164 , and 91850204)

\section{Competing interests}

The authors declare no competing financial interests. 